\title{
The Birmingham Histopathology Data Pool: a co-operative project among 10 laboratories
}

\author{
B. W. CODLING, M. K. AlEXANDER, R. G. F. PARKER, AND R. C. CURRAN \\ From the Department of Pathology, the Medical School, University of Birmingham, the South \\ Warwickshire Group of Hospitals, and the East Birmingham Hospital, UK
}

SUmmary In 1970 the Birmingham Histopathology Data Pool was established to collect information on biopsies of surgical specimens from three hospitals. The scheme has gradually expanded and 10 hospitals now participate. The pathologist gives each specimen a numerical code based on the Systematised Nomenclature of Pathology (SNOP). The information is processed by computer and stored on cumulative magnetic tape file. At regular intervals (at three, six, and nine months) 'printouts' listing all the information recorded within the interval are produced and a cumulative printout is issued when the information for a whole year is complete.

The data pool system has provided accurate alphabetical and SNOP diagnostic files and has simplified retrieval of data for both follow-up and research purposes. The pathologist may use the computer to retrieve and analyse information from the data pool for his own use or on behalf of clinicians, but he may use information from laboratories other than his own for research purposes and publication only with the permission of the pathologists who submitted the data.

The value of computer techniques for handling the large volumes of data generated in clinical chemistry, haematology, and bacteriology laboratories is well established (Whitby and Blair, 1970; Whitehead, 1970; Farrar et al., 1975). Similar techniques (Pratt and Thomas, 1966) have not been adopted widely by histopathology laboratories for two main reasons: (1) their output consists of verbal reports on the macroscopic and histological features of tissue specimens leading to conclusions or diagnoses which are the opinions of the pathologist; (2) the number of specimens handled is relatively small.

Of the several systems available for codifying histological lesions the Systematised Nomenclature of Pathology (SNOP)(College of American Pathologists, 1965 ) is that most widely used by histopathologists. Moreover, when the information from several laboratories is pooled the number of specimens increases greatly and the advantages of computerbased methods are obvious. Another advantage is that histopathological records are usually kept indefinitely, not only as a potential source of research material but because they contain information which may at some future date have a bearing on the diagnosis and treatment of individual patients. For these reasons we chose a computer-based

Received for publication 13 June 1977 system when we decided in 1970 to establish a histopathology data pool which would store codified diagnoses from a number of centres.

Initially data were collected from three laboratories-those serving the Queen Elizabeth Hospital, Birmingham, the East Birmingham Hospital, and the South Warwickshire Group of hospitals. The pool has now expanded to include 10 hospitals. ${ }^{1}$ In 1975, 45313 diagnoses were recorded (Table 1) from report forms (Fig. 1).

\section{Methods}

COMPUTER FACILITIES

Until November 1976 the data pool was based on the IBM 1440 computer at the University Medical School, which has a $16 \mathrm{~K}$ character core size. In addition to the computer the facilities included an IBM 1442 card reader and punch, an IBM 1443 line printer, two 7335 magnetic tape drives, and

1Other hospitals now in the data pool are the General Hospital, Birmingham (1973): the North Staffordshire Hospital Centre, Stoke; Good Hope District General Hospital, Sutton Coldfield; Birmingham Dental Hospital; the Midland Centre for Neurosurgery and Neurology, Smethwick; Staffordshire General Infirmary (1975): and Walsall Hospital (1976). 
Table 1 Number of diagnoses recorded in the histopathology data pool since its inception in 1970

\begin{tabular}{llll}
\hline Year & $\begin{array}{l}\text { No. of } \\
\text { participant } \\
\text { hospitals }\end{array}$ & $\begin{array}{l}\text { No. of } \\
\text { diagnoses }\end{array}$ & $\begin{array}{l}\text { No. of } \\
\text { diagnoses } \\
\text { per month }\end{array}$ \\
\hline $1970 / 1$ & 3 & 16273 & 1084 \\
1972 & 3 & 15417 & 1285 \\
1973 & 4 & 22084 & 1840 \\
1974 & 4 & 24089 & 2007 \\
1975 & 8 & 45313 & 3776 \\
\hline
\end{tabular}

two 1311 exchangeable disk drives each with a nominal capacity of 2000000 characters. Data were stored on magnetic tape (maximum size $2400 \mathrm{ft}$ $(730 \mathrm{~m}))$ with a density of 556 bytes/in $(2.5 \mathrm{~cm})$ and and inter-record gap of 0.75 in $(19.1 \mathrm{~mm})$. This density gives about 32000 records per tape. In November 1976 the data pool was transferred to a Univac 418 III computer which has a much greater handling capacity.

INPUT OF DATA

Standard 80-character punch cards are used (Fig. 2) to record details of patient identity, specimen identity, and the pathological diagnosis, coded according to SNOP, transferred either from the report form or a listing in suitable order. Columns 1-3 of the card identify the hospital; columns 4-28 identify the patient by registration number, sex, ward, surname, forenames, and year of birth; and the specimen is identified in columns $29-39$ by its date of arrival and its laboratory number.

The consultant in clinical charge of the patient is indicated (columns 40-43) by a four-digit number derived from the West Midlands Regional Health Authority's specialty and consultant code list. The type of preparation (column 44) - that is, frozen section, paraffin section, smear, and origin at either biopsy or necropsy-is indicated by a single letter code.

The pathologist code (column 45) is also a singleletter code indicating the pathologist responsible for the report. The final 16 columns of the computer card contain the SNOP code.

Although the histopathology report forms are designed to suit the individual needs of each laboratory taking part, the information required by the data pool is in the same order on each report form (Fig. 1). This facilitates the process of card punching, which is carried out centrally in the data preparation unit of the Queen Elizabeth Medical Centre Computer Services Unit.

\section{PROCESSING}

After the cards have been punched they are checked for accuracy (that is, verified) by the card punch operators and submitted to the computer. Data are checked for accuracy within defined limits (that is, validated) by the computer (see below) and then transferred to magnetic tape files by means of programs written in Autocoder and an IBM sort utility program. The validation criteria are simple in that omissions are not allowed in fields other than the description field. Alphabetical and numerical characters must appear in the appropriate columns. To cater for patients who are not registered the designation NK (not known) in the registration number field is allowed if the wardfield is punched EXT, meaning an external case. Valid data are sorted and merged with existing data on disks. The data are then transferred to magnetic tape files which are maintained in two different formats-firstly, alphabetically (by patient surname for each hospital), and, secondly, in SNOP code order. The alphabetical and SNOP magnetic tape files are designed to be error-free within the predetermined criteria of the validation programme.

\section{OUTPUT FROM COMPUTER}

Lists of validated data are produced at weekly intervals. These indicate any records which are in error, and it is the responsibility of each laboratory to resubmit these records after correction and to check that the record is complete and up to date at

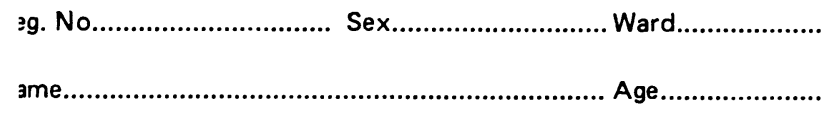

lagnosis

ature of Specimen

Fig. 1 Report form showing pattern of presentation of data to facilitate card punching. 


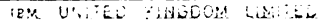

Fig. 2 Punch card routinely used by data pool.

the end of the calendar year. A print-out in laboratory number order can be used to detect the occasional omitted record. The computer routinely produces a print-out at three-monthly intervals, when each laboratory receives an alphabetical print-out of its own data in patient surname order (Fig. 3). The print-out records data in the same order as on the punch cards except that the patient's age and not year of birth is recorded. In addition, each laboratory also receives at six-monthly intervals a copy of its own data printed in SNOP diagnostic code order (Fig. 4).

At the end of each year, after a check for completeness, a final cumulative print-out is prepared for each laboratory from the alphabetical and SNOP code tapes. This acts as a permanent record in each laboratory for reference. Retrieval of data to meet individual requirements is carried out on request.

\section{Results}

COMPUTER TECHNIQUES

The techniques are relatively simple and no serious difficulties have arisen.

ACCURACY OF DATA

The application of validation criteria to the data has focused attention on the difficulties in obtaining complete and accurate information. This is par- ticularly so in the case of patient identity, and most laboratories rely entirely on the information recorded on request forms. The Queen Elizabeth Hospital, however, has an on-line hospital information system which allows checks to be made on the accuracy of patient identity. With its help assessments of the size of this problem were made.

The first test was made in June 1974 (Table 2). Of 147 patient requests which were received in one week $63 \%$ of request forms used preprinted selfadhesive identification labels and $37 \%$ were handwritten. A small number $(14 \%)$ of the self-adhesive labels were inaccurate as a result of patients having changed wards or consultant. Fifty per cent of the hand-written forms showed errors variously affecting the name, age, sex, registration number, ward, and consultant. Explanation of the need for accuracy to operating theatre staff led to a progressive reduction from $27 \%$ to $12.5 \%$ in the number of request forms showing errors over a period of six months. The error rate of $12.5 \%$ recorded in January 1975 is probably less significant than at first seems apparent since, for the purposes of this survey, a discrepancy in any one letter or digit within a field (that is, name, registration number, etc) was counted as an error.

In practice only a small proportion of errors are so serious that a report fails to reach the patient's case record. Nevertheless, in computer processing 


\begin{tabular}{|c|c|c|c|}
\hline HOSP & SURNAME & $\begin{array}{l}\text { FORE } \\
\text { NAME }\end{array}$ & SEX \\
\hline $\begin{array}{l}\text { GHH } \\
\text { GHH }\end{array}$ & $\begin{array}{l}A B 331 \\
A C K\end{array}$ & $S H$ & \\
\hline $\begin{array}{l}\text { GHH } \\
\text { GHH }\end{array}$ & $\begin{array}{l}A C K \\
A C T \text { T }\end{array}$ & $\begin{array}{l}\text { S1 } \\
\text { GA }\end{array}$ & F \\
\hline GHH & $A O A$ & MA & $F$ \\
\hline GHH & $A D A$. & MA & $\mathrm{F}$ \\
\hline GHH & $A O A$ & MA & $\mathrm{F}$ \\
\hline GHH & AOA & $B A$ & $\mathrm{~F}$ \\
\hline GHH & $A D A$ & $\mathrm{CH}$ & $M$ \\
\hline GHH & $A O A_{1}$ & EV & $\mathrm{F}$ \\
\hline GHH & $A D A$ & EV & $\mathrm{F}$ \\
\hline GHH & $\mathrm{AOA}_{1}$ & $F R$ & M \\
\hline $\begin{array}{l}\text { GHH } \\
\text { GHH }\end{array}$ & $\stackrel{A O A}{A O A_{i}}$ & JE $_{M A}$ & $F$ \\
\hline GHH & $A D A$ & RE & M \\
\hline GHH & $\hat{A D A} \mathbf{A}$ & $\hat{R E}$ & M \\
\hline GHH & $A D A$ & $v$ & $\mathrm{~F}$ \\
\hline GHH & $A D=1$ & $B E$ & $F$ \\
\hline GHH & $A O C$ & $B E$ & $F$ \\
\hline GHH & $A D C I$ & BE & $\mathrm{F}$ \\
\hline GMH & $A D E$ & MA & $F$ \\
\hline GHH & $A I R_{1}$ & $S A$ & M \\
\hline GHH & AIR & Do & $F$ \\
\hline GHH & ALBI & VE & F \\
\hline GHW & AL B & VE & $\mathrm{F}$ \\
\hline GHH & ALC & iv & $F$ \\
\hline GHH & ALD & ju & $F$ \\
\hline GHH & $A L O$ & JU & $\mathrm{F}$ \\
\hline GHH & ALD & JU & $F$ \\
\hline GHH & ALU & GE & $M$ \\
\hline GHA & ALD & $G L$ & $F$ \\
\hline GHH & ALU & JA & M \\
\hline GHH & ALD & JA & M \\
\hline GHH & $A L O$ & JA & M \\
\hline GHH & AL() & JA & $M$ \\
\hline GHH & $A L O$ & No & $F$ \\
\hline GHH & ALE & JE & $\mathrm{F}$ \\
\hline GHH & ALE. & KE & M \\
\hline GHH & ALF & vI & $\mathrm{F}$ \\
\hline $\begin{array}{l}\text { GHH } \\
\text { GHH }\end{array}$ & AL:- & $\begin{array}{l}\text { MI } \\
\text { MI }\end{array}$ & $M_{M}^{M}$ \\
\hline GHH & AL:. & $P E$ & $M$ \\
\hline GHH & ALL & PE & M \\
\hline GHH & $A L:-$. & $P E$ & $M$ \\
\hline GHH & $A L L$ & GH & $\mathrm{F}$ \\
\hline GHH & AL:- & $J E$ & $\mathrm{~F}$ \\
\hline GHH & $A L L$ & MA & $F$ \\
\hline GHH & $A L_{-}$. & $G R$ & $M$ \\
\hline GHH & ALL & $\Delta U$ & $\mathrm{~F}$ \\
\hline GHH & $A L:-1$ & Fr & $M$ \\
\hline GHH & ALL & $\begin{array}{l}G L \\
G L\end{array}$ & $\begin{array}{l}F \\
F\end{array}$ \\
\hline $\begin{array}{l}\text { GHH } \\
\text { GHH }\end{array}$ & $\begin{array}{l}\text { AL':: } \\
\text { ALL }\end{array}$ & $\begin{array}{l}G L \\
G L\end{array}$ & $\begin{array}{l}F \\
F\end{array}$ \\
\hline $\mathrm{G}, \mathrm{HH}$ & AL:- & IR & $F$ \\
\hline GHH & ALL & IR & $\mathrm{F}$ \\
\hline GHH & AL'. & IR & $F$ \\
\hline GHH & $A L L$ & JE & $\mathrm{F}$ \\
\hline GHH & $A B 3$ & SH & $F$ \\
\hline GHH & $A C K$ & SI & M \\
\hline GHH & $A C T_{1}$ & GA & $F$ \\
\hline GHH & $A D A$ & MA & $F$ \\
\hline GHH & ADA & MA & $F$ \\
\hline GHW & $\begin{array}{l}A O A \\
A O A\end{array}$ & $\begin{array}{l}\text { MA } \\
B A\end{array}$ & $\mathrm{~F}$ \\
\hline $\begin{array}{l}\text { GHH } \\
\text { GHH }\end{array}$ & $\begin{array}{l}\text { ADA } 1 \\
\text { ADA }\end{array}$ & $\mathrm{CH}$ & $F_{M}^{F}$ \\
\hline GHH & ADA & EV & $\hat{F}$ \\
\hline GHH & $A D A$ & EV & $\mathbf{F}$ \\
\hline GHH & AOA & $F R$ & $M$ \\
\hline GHH & $A D A$ & JE A & $\vec{F}$ \\
\hline GHH & $A_{10 A}$ & MA & $F$ \\
\hline Grw & $A D A$ & RE & M \\
\hline GMH & ADAI & RE & $M$ \\
\hline GrWH & $A D A$ & $\mathrm{v}$ & $\vec{F}$ \\
\hline GHH & $A D=1$ & $B E$ & $\mathrm{~F}$ \\
\hline $\begin{array}{l}\text { GHH } \\
\text { GHH }\end{array}$ & $\begin{array}{l}A O C \\
A O C 1\end{array}$ & $\begin{array}{l}B E \\
B E\end{array}$ & $F$ \\
\hline $\begin{array}{l}\text { GHH } \\
\text { GHH }\end{array}$ & $\begin{array}{l}A D C I \\
A D E\end{array}$ & $B E$ & $F$ \\
\hline $\begin{array}{l}\text { GHH } \\
\text { GHH }\end{array}$ & $\begin{array}{l}\text { ADE } \\
\text { AIRI }\end{array}$ & MA & $F$ \\
\hline $\begin{array}{l}\text { GHH } \\
\text { GHH }\end{array}$ & $\begin{array}{l}A I R I \\
\text { AIR }\end{array}$ & $\begin{array}{l}\text { SA } \\
\text { DO }\end{array}$ & $\mathbf{F}$ \\
\hline GHH & ALBI & VE & $F$ \\
\hline GHWH & $A L B$ & VE & $F$ \\
\hline GHH & ALCI & Iv & $F$ \\
\hline Grịt & ALD & ju & $F$ \\
\hline
\end{tabular}

aLPHABE IICAL REgISTER

LAB ACC TYPE ANU PATHOL- CONSUL- WARD DATE SNOP COUE DESIRIPTION

PATIENT
REG NU

$\begin{array}{ll}11 & \mathrm{~N} \\ 59 & 080291\end{array}$

$20 \quad 175232$

$\begin{array}{ll}29 & 083504 \\ 43 & 136477\end{array}$

$43 \quad 136477$

$\begin{array}{ll}40 & 075048 \\ 17 & 095669\end{array}$

$\begin{array}{ll}17 & 095669 \\ 55 & 049651\end{array}$

504965

$\begin{array}{ll}64 & 025454 \\ 31 & 069255\end{array}$

$31 \quad 162255$

$46 \quad 058383$

$\begin{array}{ll}44 & 058383 \\ 20 & 116694\end{array}$

$44 \quad 026769$

$43 \quad 026769$

$31 \quad 173903$

$\begin{array}{ll}60 & 999999 \\ 68 & 179059\end{array}$

$\begin{array}{ll}55 & 075431 \\ 55 & 075431\end{array}$

$\begin{array}{ll}55 & 075431 \\ 73 & 158005\end{array}$

$\begin{array}{ll}44 & 173997 \\ 43 & 273997\end{array}$

$\begin{array}{ll}43 & 273997 \\ 43 & 273997\end{array}$

67130237

$\begin{array}{ll}55 & 045391 \\ 55 & 176905\end{array}$

54071905

$54 \quad 171905$

54171905

$\begin{array}{ll}1 & 000663 \\ 4 & 085420\end{array}$

$\begin{array}{ll}48 & 165186 \\ 26 & 175867\end{array}$

$\begin{array}{ll}47 & 087012 \\ 28 & 173549\end{array}$

$\begin{array}{ll}28 & 173549 \\ 59 & 168704\end{array}$

$\begin{array}{ll}59 & 168704 \\ 50 & 168704\end{array}$

$\begin{array}{ll}50 & 168704 \\ 78 & 168704\end{array}$

49029492

$\begin{array}{ll}53 & 172996 \\ 23 & 183054\end{array}$

35181566

$40 \quad 181324$

49166188

$4916 h 188$

45
45089330
459330

45039330

43109586

$\begin{array}{ll}11 & \text { Nर } \\ 59 & 080291\end{array}$

$20 \quad 175232$

29083504

$\begin{array}{ll}43 & 136477 \\ 40 & 075048\end{array}$

17095669

55049651

$64 \quad 025454$

$\begin{array}{ll}31 & 069255 \\ 31 & 162255\end{array}$

$46 \quad 058383$

$\begin{array}{ll}44 & 058383 \\ 20 & 116694\end{array}$

$44 \quad 026769$

$43 \quad 026769$
43

$\begin{array}{ll}42 & 172092 \\ 31 & 173903\end{array}$

$\begin{array}{ll}31 & 173903 \\ 60 & 999999\end{array}$

$\begin{array}{ll}60 & 999999 \\ 68 & 179059\end{array}$

$\begin{array}{ll}55 & 075431 \\ 55 & 075431\end{array}$

$\begin{array}{ll}55 & 075431 \\ 73 & 158005 \\ 44 & 173997\end{array}$

HUMBER TYPE ANO PATHOL

CONSUL -

$\begin{array}{lrllll}\text { EXT } & 1 / 12 / 75 & 0600 & 4101 & 0000 & 0000 \\ \text { TGH } & 15 / 10 / 75 & 2 Y 01 & 6972 & 0000 & 0000\end{array}$

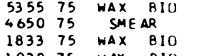

102875 WAX B10

$\begin{array}{llll}2126 & 75 & \text { WAX } & B 10 \\ 2126 & 75 & \text { WAX BIO }\end{array}$

339175 WAX BIO

$\begin{array}{llll}5459 & 75 & \text { WAX } & 810 \\ 5285 & 75 & \text { WAX } & 810\end{array}$

207675 WAX B10

312675 WAX RIU

20675 SMEAR

50475 WAX $B 10$

12075 WAX BIU

$\begin{array}{llll}120 & 75 & \text { WAX BIU } \\ 497 & 75 & \text { WAX BIO }\end{array}$

144875 WAX BIO

428575 SMEAR

521775 WAX 810

521775 WAX BIO

426275 WAX 810

$\begin{array}{llll}4804 & 75 & \text { WAX } & B 10 \\ 804 & 75 \\ 4 A X & B 10\end{array}$

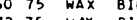

334475 HAX BIO

333875 WAX BIU

47875 WAX BIO

$\begin{array}{llll}3178 & 75 & \text { WAX BIU } \\ 4423 & 75 & \text { WAX BIO }\end{array}$

360375 WAX BIO

236775 WAX BIO

$\begin{array}{llll}15575 & \text { WAX BIU } \\ 244 & 75 \text { WAX BIO }\end{array}$

124475 WAX B10
341675 WAX B11

27775 WAX BIU
27735 WAX BIU

25375 WAX BIO

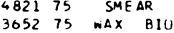

442675 WAA BIU

WAX 611

34775 WAX BIU

394875 SME AR

$\begin{array}{llll}3003 & 75 & \text { SAX F FIU } \\ 3003 & 75 & \text { WAX FIO }\end{array}$

105875 SME.AZ

196475 WAX RIII

492075 WAX RII

535575 WAX 810

465075 SMEAR

183375 HAX BIO
102875 HAX BIO

$\begin{array}{llll}1028 & 75 & \text { WAX } & 810 \\ 2126 & 75 & \text { WAX } & B 10\end{array}$

212675 WAX B10

545975 WAX BIO

528575 WAX BIO

207675 WAX BIO

312675 WAX PIO

35575 WAX BIO

20675 SMEAR

12075 WAX BIO

12075 WAX BIO

49775 WAX BIO

144875 WAX BIO

40975 WAX 810

521775 WAX B10

550975 SMEAR

3264
0098
1214
6046
6017
6017
6017
3097
6046
6046
3219

CPD 24/4/75 0100876000000000

7 TH $7 / 3 / 75 \quad 8400783100000000$

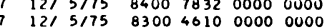

$728 / 777588400783100000000$

8 10/12/75 6600410100000000

TGH $26 / 11 / 75 \quad 8400814300000000$

G $8 / 5175 \times 900000100000000$

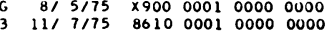

$7221 \quad 1 / 75 \quad 8400783100000000$

3097 TGH 24/ 1/75 74008131 00000000

3097 SEH $1411 / 75 \quad 7 \times 10000300000000$

$6059 \quad 7 \quad 5 / 2 / 75 \quad 8100354000000000$

3264 TGH $9 / 1 / 750401814300000000$

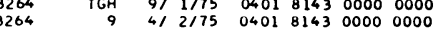

$6059 \quad 77 \quad 3 / 4 / 75 \quad 8610262000000000$

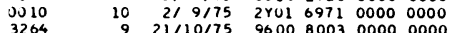

$32649921110 / 759600800300000000$

$7 \quad 24 / 11 / 75 \quad 840073810000 \quad 0000$

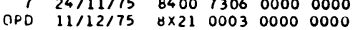

$2419 / 75 \quad 83304000 \quad 00000000$

$723 / 4 / 75 \quad 840078320000 \quad 0000$

OPD $10 / 9 / 756301400000000000$

$7 \quad 217 / 75 \quad 830073810000 \quad 0000$

$102417 / 75 \quad 4300801600000000$

DAR $31 / 1 / 75 \quad 5600000100000000$

$10 \quad 15 / 7 / 75 \quad 6200400000000000$

OPD $3 / 10 / 75 \quad 0100471200000000$

TGH $8 / 8 / 75 \quad 84007832 \quad 0000 \quad 0000$

OPD $10 / 9 / 75 \quad 0100.876000000000$

OPD $28 / 5 / 75 \quad 0401901000000000$

$9 \quad 10 / 1 / 75 \quad 780090630000 \quad 0000$

19/3/75 6600410100000000

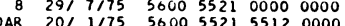

DAR $20 / 1 / 755600552155120000$

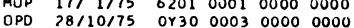

$\begin{array}{rlllll}O P D & 28 / 10 / 75 & \text { Or } 30 & 0003 & 0000 & 0000 \\ 7 & 1318 / 75 & 840078310000 & 0000\end{array}$

TGH $6 / 10 / 756600410100000000$

LVH $1 / 10 / 75$ 0401 814300000000

$0 \mathrm{PO} \quad 219 / 75 \quad 8 \times 31000300000000$

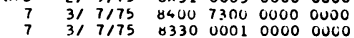

$713 / 75 \quad 3 \times 31647000000000$

$215 / 75 \quad 4300461000000000$

$\begin{array}{llllll}7 & 2 / 5 / 75 & 8400 & 7831 & 0600 & 00 j 0\end{array}$

กPD $5 / 11175$ O400 73310000 UUUO

EXT $1 / 12 / 75$ 66U0 410100000000

TGH 15/10/75 2Y01 697200000000

OPO 24747750100876000000000

$\begin{array}{crlllll} & 7 / & 3 / 75 & 8400 & 7831 & 0000 & 0000 \\ 7 & 12 / & 5 / 75 & 8400 & 7832 & 0000 & 0000\end{array}$

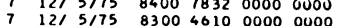

$7 \quad 28 / 7 / 75 \quad 8400783100000000$

$8 \quad 10 / 12 / 75 \quad 6600410100000000$

TGH 26/11/75 8400814300000000

$G \quad 8 / 5 / 75 \times 900000100000000$

$1117175 \quad 861000010000000$

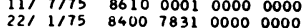

TGH 24/ 1/75 7400813100000000

SEH $\quad 14 / 1 / 75 \quad 7 \times 10000300000000$

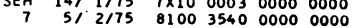

TGH $\quad 9 / 1 / 75$ 1/ 0401814300000000

9/ $1 / 75$ 0401 814300000000

$4 / 2 / 75 \quad 0401814300000000$

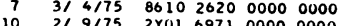

$10 \quad 2 / 9 / 75 \quad 2$ rol $69710000 \quad 0000$

$\begin{array}{llllll}9 & 21 / 10 / 75 & 9600 & 8003 & 0000 & 0000 \\ 7 & 24 / 11 / 75 & 8400 & 7381 & 0000 & 0000\end{array}$

$\begin{array}{llllll}7 & 24 / 11 / 75 & 8400 & 7381 & 0000 & 0000 \\ 7 & 24 / 11 / 75 & 8400 & 7306 & 0000 & 0000\end{array}$

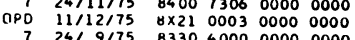

426275 WAX B10 W 6059

$7 \quad 2419 / 75 \quad 833040000000 \quad 0000$

Fig. 3 Alphabetical print-out. Occasional discrepancies appear between laboratory number and patient age-for example, patient ADA-RE, age 46 and 44, laboratory numbers 355 and 206. These records refer to two specimens, one a biopsy the other cytology, from the same patient. The error in age arose on the request form. (The latter part of patient surnames is obliterated to preserve confidentiality.)

all errors are significant, since a difference in any one digit in patient identity means that the record is regarded as being from a different patient.

Accurate identification of specimens is easier than patient identification since it is under the direct control of the laboratory. Errors are therefore rare and when they occur they are caused either by transposition of two numbers or by incorrect 


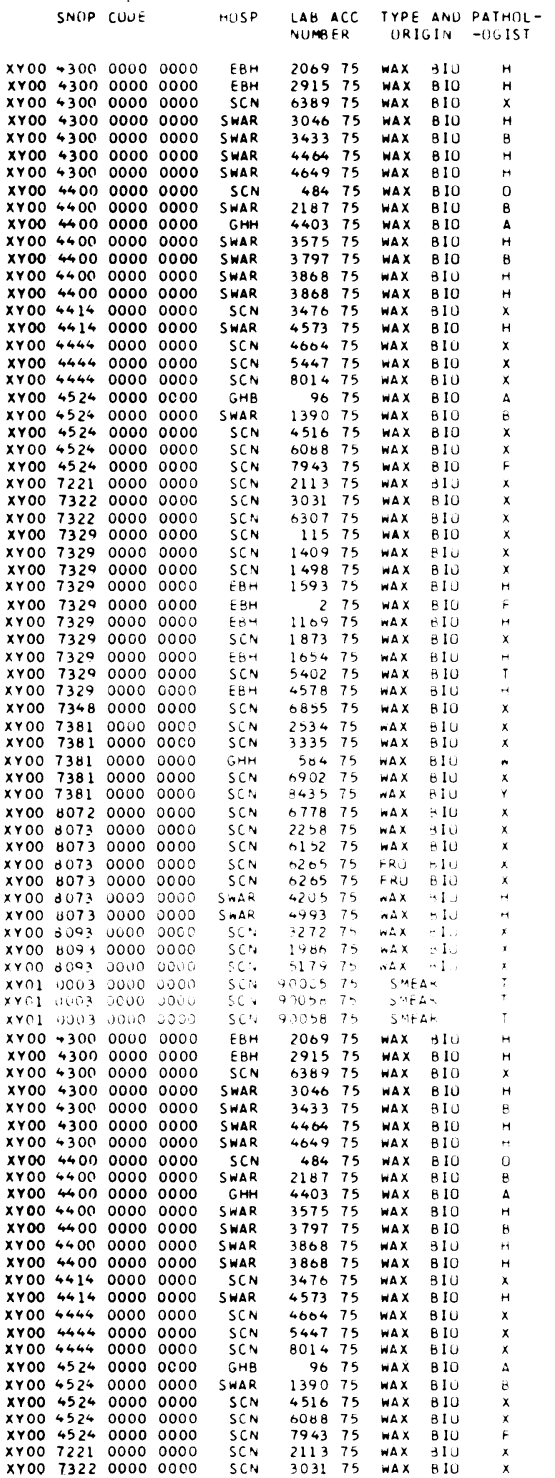

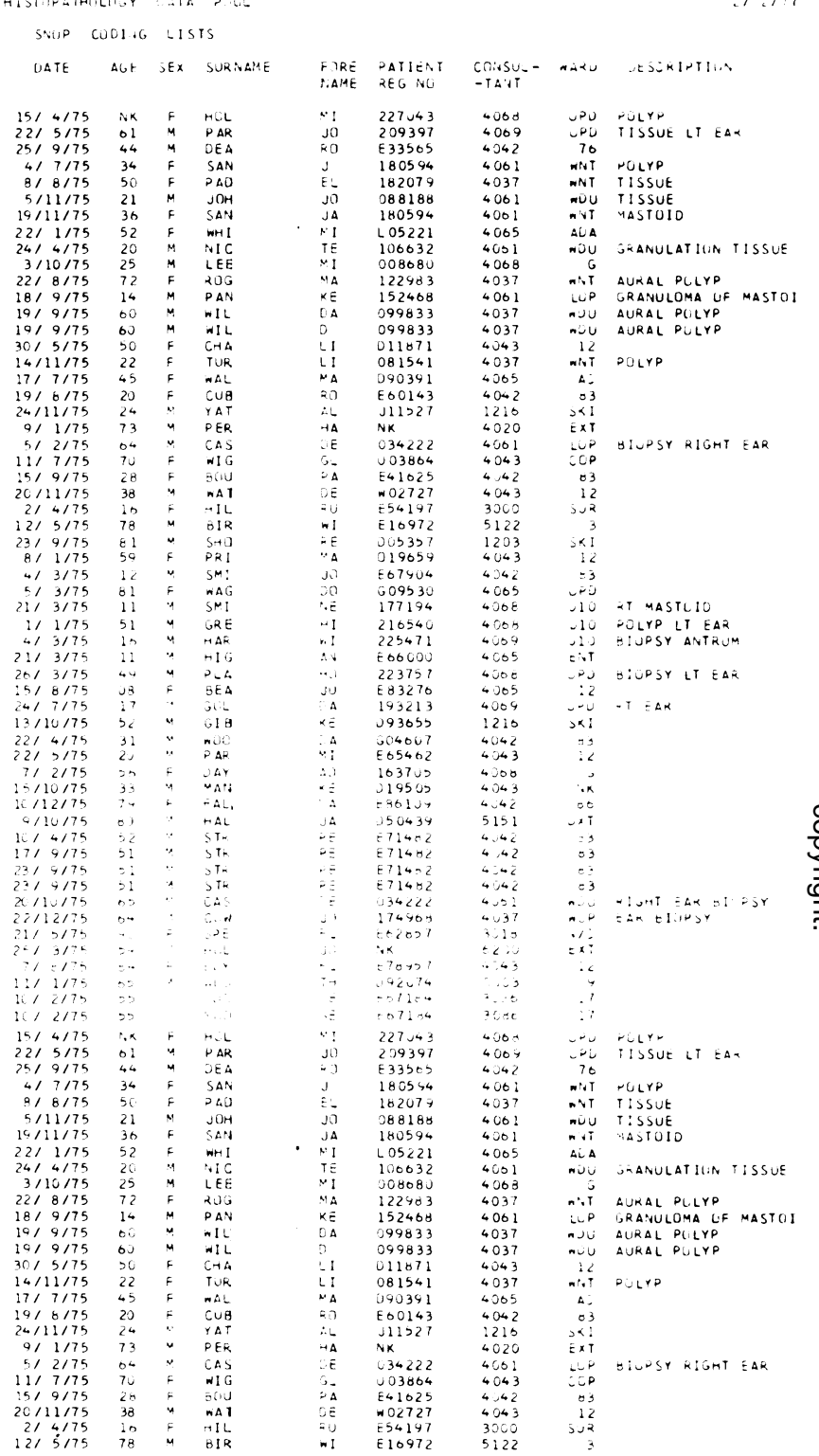

Fig. 4 SNOP diagnostic code order print-out. (The latter part of patient surnames is obliterated to preserve confidentiality.)

copying from the request form to the report form. These errors, while not unimportant, are less vital than errors in patient identity, since the most important aspect of the record is the ability to couple the correct diagnosis with the appropriate patient.

A further source of error arises from the structure of the SNOP code and the way in which codes are allocated. The code consists of four fields-Topography (T), Morphology (M), Aetiology (E), and Function $(F)$ - each having four digits. Each field has a branching structure with finer subdivisions in the field leading to a specific meaning when all four digits are used-for example, M8143 denotes 
Table 2 Survey of errors in patient identity as recorded on request forms and effect of explaining need for accuracy to operating theatre staff

\begin{tabular}{llllll}
\hline Month/year & $\begin{array}{l}\text { Sample } \\
\text { size }\end{array}$ & $\begin{array}{l}\text { No of } \\
\text { errors }\end{array}$ & $\begin{array}{l}\text { No. of } \\
\text { forms } \\
\text { affected }\end{array}$ & $\begin{array}{l}\% \text { of } \\
\text { forms } \\
\text { affected }\end{array}$ & $\begin{array}{l}\% \text { of } \\
\text { forms with } \\
\text { label }\end{array}$ \\
\hline $\begin{array}{l}\text { June } 1974 \\
\text { November }\end{array}$ & 147 & 48 & 40 & 27 & 63 \\
$\begin{array}{c}\text { 1974 } \\
\text { January 1975 } 169\end{array}$ & 34 & 24 & 21 & 72 \\
\hline
\end{tabular}

Table 3 Errors in coding and their origin detected in data retrieved for other purposes

\begin{tabular}{lccccc}
\hline & \multicolumn{2}{l}{1971} & & \multicolumn{2}{l}{1975} \\
\cline { 2 - 3 } \cline { 5 - 6 } \cline { 5 - 6 } & Number & Percentage & & Number & Percentage \\
\hline No. of diagnoses & 304 & 100 & & 327 & 100 \\
Typographical errors & 3 & 0.99 & & 2 & 0.61 \\
Pathologist coding errors & 9 & 2.96 & & - & - \\
Coding discrepancies & 7 & 2.3 & & 12 & 3.67 \\
\hline
\end{tabular}

primary adenocarcinoma. Three main types of coding errors were found during periodic assessments of retrieved data (Table 3): (1) typographical errors $(1 \%)$ due to misreading of poor handwriting and the transposition of digits; (2) miscoding by the pathologist $(0-3 \%)$ either through misreading a code or failing to remember a familiar codecorrectly; and (3) the SNOP system allows a lesion to be coded in more than one way and a pathologist may allocate a code which is different from that allocated by another pathologist for the same lesion $(2-4 \%)$.
Inflammatory lesions are particularly prone to the type of problem stated in (3). It is minimised by the use of a bench 'visual aid'.

Usually the $T$ and $M$ fields are adequate for coding a given diagnosis and a remarkably small proportion of the many thousands of codes available in the large coding manual is sufficient for everyday use. The SNOP visual aid, which is a synopsis of commonly used codes, is available but it did not entirely suit our needs. Therefore in 1971 we compiled our own. This included topographic and morphology codes along with a small number of codes to denote aetiology and function. Of the 842 morphology codes in the visual aid $50 \%$ were sufficient to provide $98 \%$ of diagnoses and the pathologist's workload in coding his diagnosis was correspondingly reduced. The Royal College of Pathologists (1975) has produced a similar visual aid designed to meet the needs of histopathology laboratories (Systematised Nomenclature of Pathology: A Bench Synopsis). The use of a visual aid encourages uniformity of coding since the choice of codes applicable to a particular lesion is much more restricted.

\section{RETRIEVAL}

Data can be readily retrieved from the routine print-out when the requirement is straightforward, but when more complex retrieval and analyses are required the computer is used. The cumulative printout provides a means of rapid retrieval of data about individual patients for follow-up and review. In addition, retrieval of significant numbers of a par-

Table 4 Topographic distribution by age of cutaneous squamous carcinoma from the Birmingham Histopathology Data Pool, 1971-76

\begin{tabular}{|c|c|c|c|c|c|c|c|c|c|c|c|}
\hline \multirow[t]{2}{*}{ Site } & \multicolumn{10}{|c|}{ Age (years) } & \\
\hline & $\leqslant 9$ & $10-19$ & $20-29$ & $30-39$ & $40-49$ & $50-59$ & $60-69$ & $70-79$ & $80-89$ & $\geqslant 90$ & \\
\hline Scalp & - & - & - & - & 1 & 3 & 4 & 3 & 4 & - & 15 \\
\hline Forehead & - & 一 & 一 & - & - & - & 1 & 1 & - & - & 2 \\
\hline Face & - & - & - & - & 1 & 12 & 20 & 22 & 17 & 1 & 73 \\
\hline Nose & - & - & - & - & - & 1 & 1 & 1 & 1 & - & 4 \\
\hline Lip & - & - & - & - & - & $i$ & 3 & - & - & - & 4 \\
\hline Ear & - & - & - & - & - & $i$ & 15 & 8 & 6 & 2 & 32 \\
\hline Neck & - & - & - & - & - & 1 & 4 & 2 & 1 & - & 9 \\
\hline Shoulder & - & - & - & - & - & - & - & - & - & - & - \\
\hline Arm & - & - & - & - & - & 2 & 2 & 3 & 2 & 1 & 10 \\
\hline Hand & - & - & - & - & - & 2 & 13 & 14 & 9 & - & 38 \\
\hline Chest & - & - & 一 & - & - & - & 1 & 1 & 1 & - & 3 \\
\hline Back & - & - & - & - & - & - & 1 & - & - & - & 1 \\
\hline Abdomen & - & - & - & - & - & - & - & 1 & - & - & 1 \\
\hline Vulva & - & - & - & - & 1 & - & 1 & 2 & - & 1 & 5 \\
\hline Scrotum & - & - & - & - & - & 2 & 3 & - & - & - & 5 \\
\hline Anus & 一 & - & - & 1 & 1 & 7 & 13 & 1 & 2 & 1 & 26 \\
\hline Thigh & - & - & - & $i$ & - & - & 2 & 2 & 3 & - & 8 \\
\hline Knee & - & - & - & - & 1 & - & 1 & - & 1 & - & 3 \\
\hline Lower leg & - & - & 1 & - & 1 & 1 & 11 & 9 & 3 & 1 & 27 \\
\hline Foot & - & - & - & - & - & - & 3 & - & - & - & 3 \\
\hline Totals & - & - & 1 & 2 & 6 & 33 & 99 & 70 & 50 & 7 & 269 \\
\hline
\end{tabular}


ticular lesion occurring at a single anatomical site is possible from the SNOP ordered file. This is of particular value not only for teaching but also for purposes such as reviewing the histological features of a lesion when establishing a diagnosis is difficult or testing a new staining technique on existing material.

Retrieval from the computer is done at the request of the pathologist either for his own purposes or on behalf of clinicians. A request form stating the information required is sent to the computer department, and the most suitable method of retrieving the information required may be discussed with the pathologist to ensure accuracy of retrieval. The following three major types of retrieval have been used.

(1) Searches on the basis of a single code or group of codes. For the pathologist this usually means retrieval of a lesion occurring at a number of different anatomical sites, something that is difficult with a manual system. Similarly, a group of cases of interest to a particular clinician can be retrieved on the bases of the consultant code and appropriate SNOP code.

(2) Detailed analysis of large volumes of data. This may be illustrated by reference to specific examples.

Table 5 Comparisons in diagnosis of frozen sections and paraffin sections in 482 diagnoses of 424 frozen sections from the Queen Elizabeth Hospital, October 1970 to December 1971

Diagnosis*

\begin{tabular}{ll}
\hline Frozen section & Paraffin section \\
\hline Breast duct papilloma & Fibroepithelial hyperplasia \\
Breast fibroadenoma (2 cases) & Fibroepithelial hyperplasia \\
Breast sclerosing adenosis & Carcinoma \\
Lymph node plasmacytoma & Inflammatory reaction \\
Parathyroid, Abnormal (2 cases) & Adenoma \\
\hline
\end{tabular}

Diagnosis modified in 35 cases and changed in seven.

Table 6 Modifications added to the system in the light of previous limitations

\begin{tabular}{ll}
\hline Card column & Future usage \\
\hline $57-60$ & $\begin{array}{l}\text { User codes: } 4 \text { characters-information of } \\
\text { interest to individual laboratory recorded without } \\
\text { reference to other users } \\
\text { Single character code denoting specimens of } \\
\text { particular interest } 1=\text { photographed, } 2= \\
\text { preserved, } 3=\text { good teaching case. } 4=1 \text { and } 2, \\
5=1 \text { and } 3,6=2+3,7=1 \text { and } 2 \text { and } 3\end{array}$ \\
62 & $\begin{array}{l}\text { Single character code indicating wish to follow up } \\
\text { later after appropriate reminder from computer } \\
\text { Single character code allowing pathologist to } \\
\text { grade a lesion } \\
\text { Single character code indicating provisional } \\
\text { (1) or uncertain (2) diagnosis. } 3=1 \text { and } 2\end{array}$ \\
\hline
\end{tabular}

(a) The frequency with which the malignant form of pleomorphic salivary tumour was diagnosed relative to other salivary gland tumours was investigated and found to be $6.5 \%$-greater than expected (Evans and Cruikshank, 1970). Examination of the data showed that the incidence was in fact no greater but that some pathologists had tended to code as malignant a number of recurrent tumours which were not frankly malignant but showed suspect histological and cytological features. This was probably an inappropriate use of the code in the strictest sense, but it illustrates the importance of the pathologist coding his own diagnoses. In doing so he allocates the code which he considers most appropriate, whereas if he delegates the task to non-medical personnel he has to rely on their ? interpretation of his findings and thereby increases the margin for error.

(b) By plotting scattergrams of patients' ages 은 against the anatomical site of a lesion (Alexander, personal communication 1975) atypical cases may be identified. In the case of squamous carcinoma of skin a scatter of cases was found (Table 4) with most lesions located in the upper part of the body in older patients. Lesions which fall outside the main grouping are worthy of further investigation if order to assess the accuracy of the initial diagnosis or the presence of other predisposing factors such as industrial exposure.

(c) The sex and age distribution of different morphological lesions occurring at a single anatomical site can be compared and patterns worthy of further study may emerge. For example, the age $\overline{\bar{O}}$ distribution in men for histologically normal appendices was identical with that for acutely inflamed appendices whereas in women two distinct patterns were found, presumably because a normal appendix is sometimes removed from a woman at laparotomy for abdominal pain due to gynaecological causes. This finding of a similar pepulation distribution in the male might represent disease at a stage before the development of overt acute appendicitis as assessed by conventional histological criteria.

(3) Data from a particular laboratory can be retrieved and analysed for patterns of work. For N example, the number of patient requests submitted for frozen section, cytological examination, and routine paraffin section examination can be determined and, by reference to the source of the request and the final diagnosis, the pattern of the workload of a laboratory in relation to the source and type of work performed can be determined. This approach offers an easy method of comparing frozen section and paraffin section diagnoses (Table 5). Moreover, retrieval of cytology and subsequent biopsy for 
both comparative and follow-up purposes is facilitated since all records for a particular patient within a single year are located together within the alphabetical file.

\section{CONFIDENTIALITY}

The diagnostic information in computer print-outs and on the magnetic tapes is in code and unintelligible without knowledge of the coding system and access to coding manuals. Consequently the use of a computer does not increase the problem of confidentiality which already exists with the longterm siorage of reports in laboratories and their use throughout hospitals and cancer registries. When a pathologist wishes to restrict the information various parts of the record can be deleted as it is printed from the magnetic tape files.

\section{FUTURE DEVELOPMENT}

In November 1976 the system was transferred to a Univac 418-III computer. This machine's greater capacity has several advantages, the most important of which is the ability to maintain all data for a whole year on a single file in alphabetical order from which a print-out in any predetermined order can be prepared. More rapid retrieval of data and more complex analyses are also possible. The change from one machine to another meant extensive reprogramming, which led to a critical review of the system and to improvements. The most important of these concern the specimen description fields, columns 46-64 on the punch card (Table 6). Further modifications affect the output of data. The validation listings will be printed monthly instead of weekly in alphabetical order in order to supplement the three-monthly alphabetical print-out.

Since the data pool records more than 40000 diagnoses a year the physical size of print-out of the SNOP diagnostic file is too large for easy handling and it will be limited to the information from individual laboratories in the same way as the alphabetical listing. Finally, the transfer to the Univac 418-III computer means that the capacity of the computer is no longer a constraint and the data pool can now expand more rapidly. Experience suggests that a data pool serving all laboratories in a region is feasible and would have many advantages over a small pool.

\section{Discussion}

Since its inception in 1970 the Birmingham Histopathology Data Pool has acquired a large volume of data. Significant numbers of a range of uncommon lesions are now on file and available for comparison and research purposes. The system provides an efficient method of maintaining index files in patient name (alphabetical) and disease (SNOP code) order, and the printed version of the files containing the information from individual laboratories provides an easy-to-use reference system giving access to information about specimens examined in the various laboratories. The data concerning cases of a particular lesion can be retrieved and analysed within defined limits. These data can be sorted into any order so that similar cases or specific features of one type of case come together and the task of the individual who has requested the retrieval is simplified.

Our experience with the computer-based data pool contrasts favourably with the experience of operating indexes and laboratory report files maintained in traditional ways. The latter generally consists of patient name and disease indexes and use copies of reports filed in laboratory number. They often work well for short-term reference but as information accumulates over the years more and more effort is required to search this type of file. Moreover, the information retrieved is incomplete. In the computer-based system the laboratory report files are maintained as in manual systems but the patient name and disease indexes are maintained by the computer.

The disease index can be more detailed than in a manually operated system since the problems associated with retrieval from manual systems are insignificant. Our experience also shows that files maintained on the computer are more accurate than manually maintained files because the computer program checks for accuracy within the predetermined criteria of the validation program and rejects records which it considers to be inaccurate or incomplete.

Computer retrieval and analysis of groups of cases is invaluable in establishing the incidence of various conditions within different areas, and thus in supplying important epidemiological information for all types of lesions similar to that already provided for neoplasms by cancer registries. The potential of this aspect will be fully realised only when the majority of hospitals in a region submit data.

A variety of other uses are possible-for example, the diagnostic habits of a laboratory can be determined and compared with those of other laboratories in the whole data pool. Thus, by monitoring the work performed by a laboratory and observing any change or trends, the pathologist can use this approach as a form of quality control or quality assurance. This approach to quality assurance has hardly been explored so far but promises to be of greater value to the practising histopathologist than 
current methods, which are usually based on his performance in diagnosing a series of difficult test sections, often prepared in an unfamiliar way and taken out of their clinical context. As part of the same monitoring process the effectiveness of different diagnostic techniques such as frozen sections versus paraffin sections may be compared.

Occasional discrepancies occur in the choice of codes for similar lesions between laboratories and between individual pathologists, partly because of the wide range of codes available to the pathologists in the complete SNOP manual. The use of a more limited range of agreed codes in the bench visual aid has helped to make the choice of the code for a particular lesion more consistent. Greater uniformity of coding could also be achieved either by a team of suitably trained coding clerks or by using an automatic coding system such as that developed by Coles and Slavin (1976). The use of non-medical personnel, however, adds to the expense and increases the risk of serious errors in coding through misinterpretation of words or phrases in a report. The alternative of automatic coding from free-text diagnosis requires diagnoses to be in a standard SNOP format. Since such a system is not yet fully developed a proportion of diagnoses have to be coded manually. Until this type of system has been fully developed and evaluated the practice in the Birmingham scheme will be for the pathologist to allocate the code considered most appropriate on the basis of the histological features.

Ten hospitals contribute to the Birmingham Histopathology Data Pool. We hope that the benefits of participation will encourage others to join. The number of hospitals that can usefully contribute to a data pool is virtually unlimited in terms of the computer capacity available, but for administrative reasons the most suitable size would seem to be one health region. This has the addedo advantage of being the area covered by most regional cancer registries.

We thank the staff of the Queen Elizabeth Medical Centre Computer Unit, in particular S. Sargent, chief systems analyst, and B. F. Scott, manager of $\stackrel{\mathbb{Q}}{\varrho}$ the IBM 1440 computer, who have been responsible for the computer aspects of this project. We also $\rightarrow$ thank the many pathologists working in the $10^{\circ}$ centres contributing to the data pool for their co- $\vec{\omega}$ operation and interest.

\section{References}

Coles, E. C., and Slavin, G. (1976). An evaluation of $\vec{N}$ automatic coding of surgical pathology reports. Journal of Clinical Pathology, 29, 621-625.

College of American Pathologists, Committee on Nomenclature and Classification of Disease (1965). System- $\mathrm{S}$ atized Nomenclature of Pathology. Chicago.

Evans, R. W., and Cruickshank, A. H. (1970). Epithelial Tumours of the Salivary Glands. Saunders, Philadelphia.

Farrar, J. L., Brogan, T. D., Moulding, T., Taylor, R..Э and Page, M. (1975). Use of a computer for producing microbiological reports and for data storage and processing. Journal of Clinical Pathology, 28, 580-5 $\overrightarrow{0}$

Pratt, A. W., and Thomas, L. B. (1966). An informati processing system for pathology data. Pathology Annu $1,1-21$.

Royal College of Pathologists (1975). Systematised Nomenclature of Pathology: A Bench Synopsis.

Whitby, J., and Blair, J. N. (1970). A computer-linked气̊ data processing system for routine hospital bacteri- $\varrho$ ology. In Automation, Mechanization and Data $\overrightarrow{\vec{F}}$ Handling in Microbiology, edited by A. Baillie and $\frac{0}{3}$ R. J. Gilbert, pp. 23-32. Academic Press, London and New York.

Whitehead, T. P. (1970). Automated clinical chemistry (Abstract). Proceedings of the Nutrition Society, 29, 106. 